\title{
Diseño Conceptual de un Cohete de Tres Etapas Para Transportar Una Carga Útil De 200 kg A Una Órbita Baja
}

\author{
CONCEPTUAL DESIGN OF A THREE-STAGE ROCKET TO TRANSPORT A LOAD OF 200 \\ kg TO A LOW ORBIT
}

\author{
Daimer Ospina Contreras \\ ${ }^{1}$ Instituto Nacional de pesquisas Espaciais. \\ ${ }^{2}$ Programa de Ingeniería Aeronáutica, \\ Universidad San Buenaventura. \\ Correspondence \\ Daimer Ospina Contreras, Instituto \\ Nacional de pesquisas Espaciais \\ Email:ingdaimerospina@hotmail.com \\ Luis Carlos Roldan Torres, Instituto \\ Nacional de Pesquisas Espaciais \\ Email: luis.roldant@outlook.com
}

Copyright : Licencia de Creative Commons Reconocimiento-NoComercial 4.0 Interna.

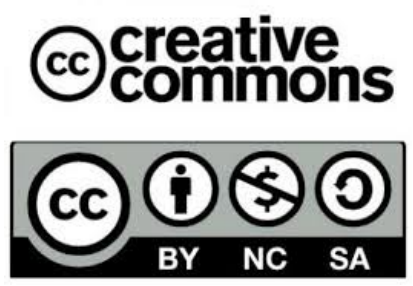

The publication of this journal is funded by Universidad ECCI, Bogotá-Colombia.

Editors: Robert Paul Salazar, Germán Chaparro.

Editorial assistant : Luz Adriana Suáres Suáres.

How to cite

Daimer Ospina, Luis Roldán .,

CONCEPTUAL DESIGN OF A

THREE-STAGE ROCKET TO TRANSPORT

A LOAD OF $200 \mathrm{~kg}$ TO A LOW ORBIT,

TECCIENCIA, Vol. 15, No. 29, 1-8, 2020

DOI:http://dx.doi.org/10.18180/tecciencia.2020.29.1

\section{Luis Carlos Roldán Torres (D) 1,2*}

ABSTRACT. This article presents the conceptual design of a three-stage rocket to carry $200 \mathrm{~kg}$ payload to low Earth orbits (by the acronym LEO). From a case study of the flight path of a three-stage rocket with solid propellants, proceed to select the propellant mixture that meets the design requirements and path raised flight, taking into account that it can produce nationwide. The propellant thermochemical behavior is obtained with CPROPEP software. Knowing the values of pressure, temperature and specific heat ratio in the combustion chamber proceeds to design rocket engines to ensure total velocity change in the system equivalent to those obtained in the analysis of the flight path. With the design of the propulsion duct materials that withstand the thermal loads and pressure, to give way to structural design giving an approximation of carrier rocket are chosen.

keywords: Rocket, rocket engine, low earth orbit, propellant.

RESUMEN. El presente artículo plantea el diseño conceptual de un cohete de tres etapas para transportar carga útil de $200 \mathrm{~kg}$ a orbitas bajas de la Tierra (por su sigla en inglés LEO). A partir de un caso de estudio de la trayectoria de vuelo de un cohete de tres etapas con propelentes sólidos, se procede a seleccionar la mezcla de propelente que se ajuste a los requerimientos del diseño y trayectoria de vuelo planteada, teniendo en cuenta que se pueda producir a nivel nacional. Conociendo los valores de la presión, temperatura y relación de calores específicos en la cámara de combustión se procede a diseñar los motores cohete para garantizar un cambio de velocidad total en el sistema, equivalente a los obtenidos en el análisis de la trayectoria de vuelo. Con el dimensionamiento de los ductos propulsivos se eligen los materiales que soporten las cargas térmicas y de presión, para dar paso al diseño estructural dando una aproximación del cohete portador.

Palabras clave: Cohete, empuje, motor cohete, órbita baja de la Tierra (LEO), propelente.

*Equally contributing authors. 


\section{1 | INTRODUCTION}

Durante años la humanidad ha soñado con explorar el espacio y por ello se da la elaboración de cohetes espaciales como el medio de transporte de distintos tipos de carga útil; estos cohetes son propulsados mediante el uso de diversos tipos de propelentes y generalmente están seccionados en varias partes llamadas etapas. Este tema ha sido muy renombrado desde la década de los años 50, sin embargo, el reto de querer ir a la Luna y a otros cuerpos celestes se remonta muchos años atrás con la carrera espacial que había entre los estadounidenses y los rusos [1]

Países como Argentina y Brasil tienen una rica historia en aportes para la tecnología aeroespacial en Latinoamérica. Las contribuciones más importantes fueron generadas por las instituciones, condiciones económicas y políticas de los gobiernos además de los acuerdos establecidos para promover la investigación en este campo. Estos dos países son buenos ejemplos de que en Latinoamérica se pueden realizar grandes aportes, y se resalta la importancia de la colaboración entre el gobierno y las diferentes instituciones académicas para el desarrollo del proceso investigativo. Gracias al apoyo de instituciones privadas y del estado se ha logrado incrementar los desarrollos tecnológicos en el campo aeroespacial, y se ha promovido la investigación obteniendo importantes avances para el sector convirtiéndose en la base para estudios posteriores. La falta de interés causa impedimento para seguir avanzando en el descubrimiento de datos relevantes, lo cual hace que los países no puedan emerger tecnológicamente como una potencia y se queden en la retaguardia de sus colegas. (Gadzheva, 2008).

A nivel institucional se cuenta con los trabajos de grado en el área de diseño conceptual y preliminar de un cohete portador de dos etapas para el transporte de carga útil desde la superficie de la Tierra a orbitas bajas, así como también se cuenta con avances en el diseño de motores cohetes de propelente sólido y el diseño de motores cohete iónicos junto con el estudio de su respectivo comportamiento dinámico para aplicaciones espaciales en maniobras de control y guía de satélites (Calderon \& Murcia 2009; Escorcia, 2011; Robledo 2011).

El siguiente artículo presenta el diseño conceptual de un cohete de tres etapas con propelente sólido capaz de transportar una carga útil de $200 \mathrm{~kg}$ a órbitas bajas de la Tierra. Se presenta el dimensionamiento, las características técnicas, la selección del material a utilizar en cada una de las etapas y de los componentes del propelente empleado, dando seguimiento a la tesis de maestría de la Universidad Nacional de Colombia titulada "Estudio de la trayectoria de un cohete de tres etapas lanzado desde el territorio Colombiano" debido a que esta plantea los estudios realizados para el lanzamiento de cohetes desde el territorio colombiano, condiciones aerodinámicas, dinámicas y de control que afectan este tipo de vehículos [2, 3, 4, 5] apoyando el desarrollo de investigaciones en el campo aeroespacial para que así surja en un futuro cercano una industria representativa en el ámbito del espacio en el territorio colombiano.

\section{2 | PARÁMETROS DE DISEÑO}

Para empezar con el diseño es importante encontrar los problemas a solucionar y las necesidades que se quieren satisfacer al realizar el modelo, por esta razón el cohete será diseñado mediante los siguientes parámetros y restricciones:

- Las cámaras de combustión de los motores cohete deberán resistir presiones dadas entre 25 Atm y 50 Atm que son las proporcionadas por diseño para que el cohete alcance las velocidades y empujes requeridos en cada etapa.

- Los motores cohete deben ser fabricados en un material liviano que pueda ser elaborado en Colombia.

- El cohete debe ser capaz de transportar una carga útil de $200 \mathrm{~kg}$ a una órbita baja de la Tierra.

Teniendo en cuenta unos parámetros iniciales tomados tanto de estudios anteriores como dados por los autores se procede a diseñar los motores cohete para cumplir con los parámetros iniciales. 


\section{3 | SELECCIÓN DEL PROPELENTE}

Para empezar a diseñar los motores cohete es importante tener claro qué clase de propelente se va a usar. Las características del mismo determinan las dimensiones de cada uno de los motores cohete para las diferentes etapas. El propelente sólido a usar en cada uno de los motores cohete será la combinación de óxido de aluminio (Al), hidroxilo polibutadieno terminado (HTPB) y perclorato de amonio (Ap), el perclorato de amonio y el nitrato de amonio es usado para las primeras etapas en los cohetes de la NASA, los Ariane y algunos cohetes chinos. Actualmente en Brasil se está trabajando con este tipo de propelentes debido a que los cohetes como el VSB que fue diseñado principalmente con propelente a base de perclorato de amonio. La industria militar colombiana INDUMIL es la única empresa estatal que trabaja en la producción de propelentes sólidos y que cuenta con los permisos requeridos para esta, produciendo propelentes de doble base compuestos principalmente de amonio o nitrato de potasio, haciendo posible la elaboración del propelente propuesto dentro del territorio colombiano [6].

Como concepto es importante determinar cuál es la característica del propelente al ser quemado y la propulsión de cada uno de los motores cohete en cada etapa. Para ello se emplea un software creado por la NASA llamado CPROPEP que fue elaborado mediante una investigación realizada por la NASA acerca del comportamiento de los propelentes y del flujo en las zonas de la cámara de combustión, garganta y tobera propulsiva. Se realizan las simulaciones de combustión del propelente para obtener la mezcla ideal de cada uno de los componentes químicos buscando un impulso específico óptimo para los motores. Se toma una muestra de 100 gramos que representan el $100 \%$ donde se determina que la mezcla de propelente ideal es de, $80 \% \mathrm{Ap}$, $10 \%$ HTPB y $10 \%$ Al, debido a que genera un impulso especifico por cada gramo de propelente (ISP/g) aproximadamente de $219,836 \mathrm{~s}$.

El factor de selección para los motores cohete es el impulso específico que depende de las propiedades termoquímicas del propelente como la temperatura, la masa molecular, relación de calores específicos $\gamma$ la constante del gas. Entre mayor sea el impulso especifico del propelente, más energía química se transforma en energía cinética, que a su vez se relaciona con la fuerza de empuje como se observa en la Eq. (1), donde el impulso específico Isp está dado en función de la fuerza de empuje $F$, la gravedad $g_{o}$ y el flujo másico $d m / d t$ o en función dela velocidad del gas $v_{g}[7]$.

$$
I s p=\frac{F}{g_{o} \dot{m}}=\frac{v_{e}}{g_{o}}
$$

En las Tablas 1 y 2 se muestran las características iniciales del propelente para cada etapa. Dentro de los datos obtenidos se muestran los valores de la relación entre calores específicos $\gamma$, la temperatura al interior de la cámara $T_{o}$, la densidad de la muestra de propelente $\rho$, la constante de los gases $R$, y los calores específicos a presión y volumen constate $C_{p}$ y $C_{v}$ respectivamente.

TABLA 1 Características del propelente para la primera etapa.

\begin{tabular}{|l|l|}
\hline$\gamma$ & 1,206 \\
\hline$I s p / g(\mathrm{~s})$ & 219,836 \\
\hline$T_{o}(\mathrm{~K})$ & 2889,340 \\
\hline$\rho\left(\mathrm{kg} / \mathrm{m}^{3}\right)$ & 2153 \\
\hline$R(\mathrm{~J} / \mathrm{kgK})$ & 274,426 \\
\hline$C_{p}(\mathrm{~kJ} / \mathrm{kgK})$ & 1,613 \\
\hline$C_{v}(\mathrm{~kJ} / \mathrm{kgK})$ & 1,337 \\
\hline
\end{tabular}

TABLA 2 Características del propelente para la segunda y tercera etapa.

\begin{tabular}{|l|l|}
\hline$\gamma$ & 1,202 \\
\hline$I s p / g(\mathrm{~s})$ & 203,359 \\
\hline$T_{o}(\mathrm{~K})$ & 2846,792 \\
\hline$\rho\left(\mathrm{kg} / \mathrm{m}^{3}\right)$ & 2153 \\
\hline$R(\mathrm{~J} / \mathrm{kgK})$ & 274,426 \\
\hline$C_{p}(\mathrm{~kJ} / \mathrm{kgK})$ & 1,646 \\
\hline$C_{v}(\mathrm{~kJ} / \mathrm{kgK})$ & 1,369 \\
\hline
\end{tabular}

Como se observa el impulso específico decrece para la segunda y tercera etapa debido a que las presiones de la cámara de combustión de estas últimas etapas es de 25 Atm lo que la diferencia de la primera que es de 50 Atm [6]. En la gráfica de la Fig. 1 se ve reflejado que el impulso específico es proporcional a la presión 
aplicada en la cámara de combustión.

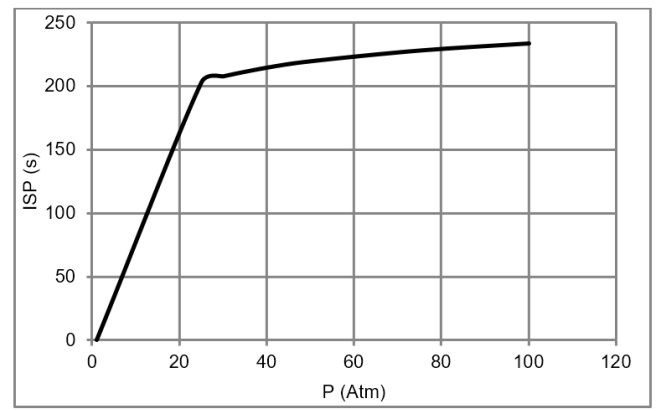

FIG. 1 Relación de impulso especifico con respecto a la presión de combustión para el propelente.
TABLA 3 Propiedades del fluido a la salida de motor cohete en primera etapa

\begin{tabular}{|l|l|}
\hline$a(\mathrm{~m} / \mathrm{s})$ & 700.01 \\
\hline$T_{e}(\mathrm{~K})$ & 1480.41 \\
$\rho_{e}\left(\mathrm{~kg} / \mathrm{m}^{3}\right)$ & 84.04 \\
$P_{e}(\mathrm{~Pa})$ & 101325 \\
$M$ & 3.03 \\
\hline$V_{e}(\mathrm{~m} / \mathrm{s})$ & 2126.93 \\
\hline
\end{tabular}

\section{4 | DISEÑO DE LOS MOTORES COHETE}

Las presiones y temperaturas del gas en la tobera se comportan idealmente de manera isoentrópica, suponiendo que no hay transferencia de calor y que el $100 \%$ de la energía térmica se convierte en energía cinética, sin perdidas por fricción de los gases con las paredes o generación de turbulencias, se aplican las Eqs. (2) y (3) para analizar la disminución de presión y temperaturas con el aumento de velocidad del gas desde la cámara de combustión hasta la zona de expansión [8].

$$
\frac{P_{c}}{P_{e}}=\left[1+\frac{\gamma-1}{2} M_{e}^{2}\right]^{\frac{\gamma}{\gamma-1}}
$$

$$
\frac{T_{c}}{T_{e}}=1+\frac{\gamma-1}{2} M_{e}^{2}
$$

Con los resultados obtenidos por el propelente se puede empezar a diseñar los ductos propulsivos de los motores cohete donde las características del fluido a la salida del motor cohete son dadas en la Tabla 3, mostrándose los valores de la velocidad del sonido a, la temperatura $T_{e}$, densidad $\rho_{e}$, presión $P_{e}$, y velocidad $V_{e}$.

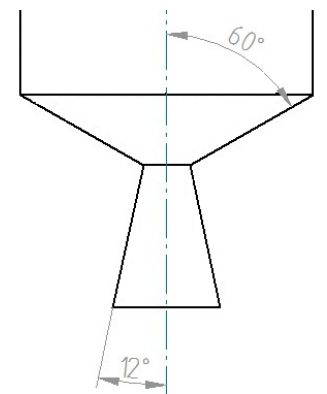

FIG. 2 Ángulo de convergencia y divergencia para los motores cohete.

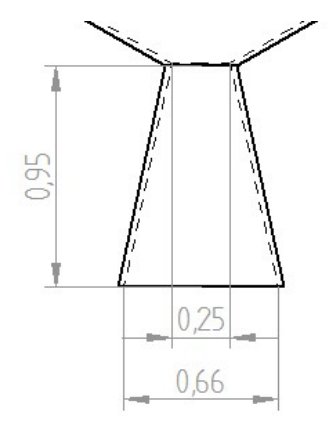

ETAPAI

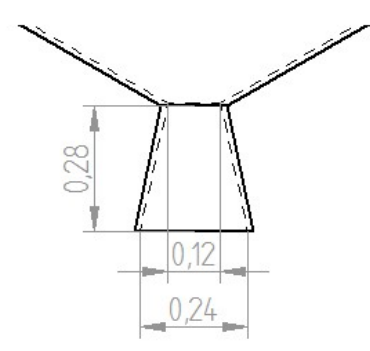

ETAPA II

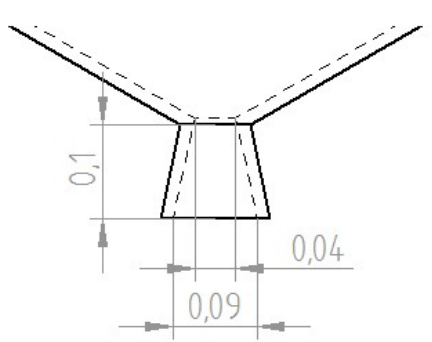

ETAPA III

FIG. 3 Dimensiones de las toberas para los motores cohete de primera, segunda y tercera etapa respectivamente en metros.

Los subíndices c y e en las relaciones de presión y temperatura mostradas en las ecuaciones (2) y (3) representas los valores dentro de la cámara y a la salida de la tobera, mientras que $M$ representa la velocidad de los gases en función del número Mach. Para el dimensionamiento se tiene en cuenta el ángulo de divergencia de los motores cohete $\theta$ que para todos va a ser de $12^{\circ}$ debido a que produce una eficiencia propulsiva $\lambda$ 
aproximada de $98.9 \%$, también teniendo en cuenta que el ángulo de divergencia es de $60^{\circ}$ con respecto al eje axial del cohete [9].

$$
\lambda=\frac{1}{2}(1+\cos \theta)=0.989 \quad \text { para } \quad \theta=12^{\circ} .
$$

Con este comportamiento del fluido se dimensiona el ducto propulsivo mediante el software CAD Solidworks teniendo en cuenta que la forma y áreas de las toberas sean ideales para soportar la temperatura, presión y velocidad de salida de los gases (ver Figs. 3 y 4).

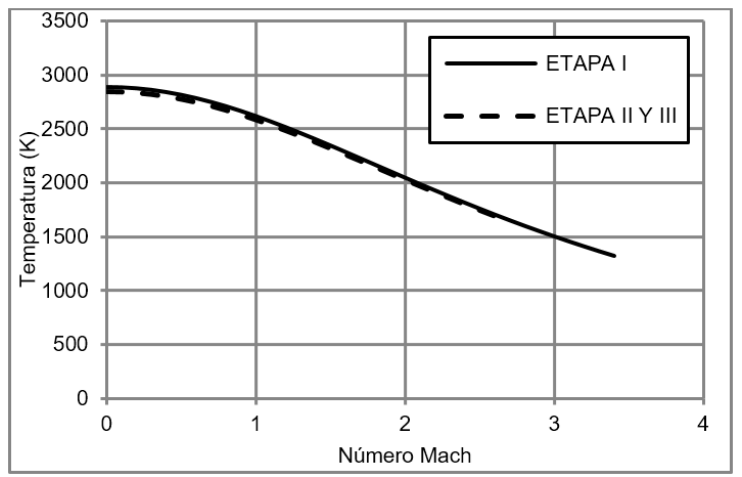

FIG. 4 Número mach vs temperatura del gas.

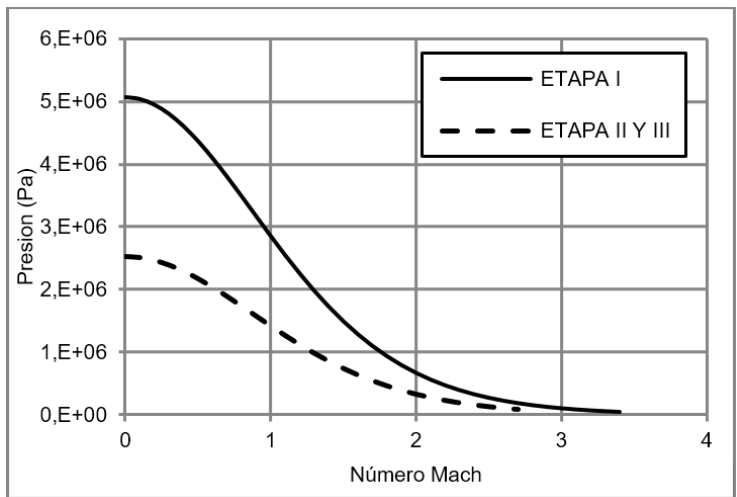

FIG. 5 Número mach vs temperatura del gas.

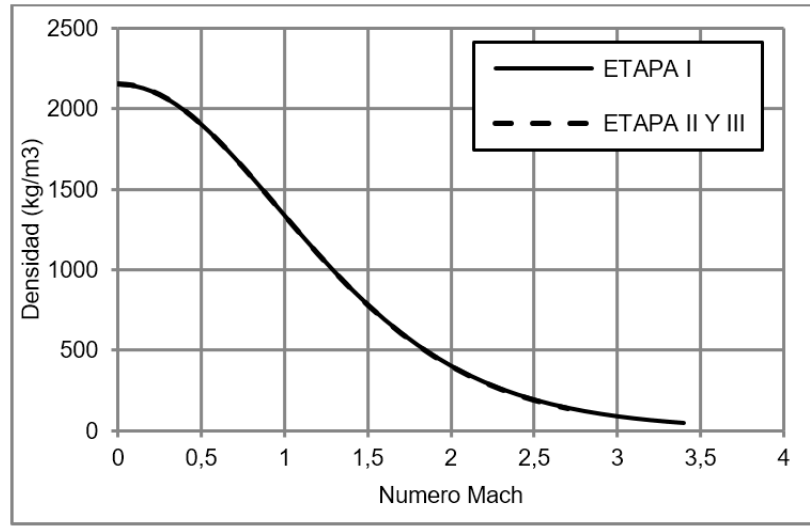

FIG. 6 Número mach vs densidad del gas.

Finalmente después de tener las dimensiones del ducto propulsivo se realiza un análisis de las propiedades del fluido con respecto a la velocidad para analizar el comportamiento de las mismas, donde se determina que cada una de las propiedades decrece a medida que aumenta la velocidad como se puede apreciar en las gráficas de las Figs. 4, 5 y 6.

\section{5 | CÁMARAS DE COMBUSTIÓN}

En la cámara de combustión de los motores cohete de propelente sólido se almacena y realiza el proceso de combustión del propelente para las diferentes etapas del cohete. Para empezar con el diseño de las cámaras de combustión de cada etapa, es importante saber a qué temperaturas y presiones va a operar. En la Tabla 4 se muestran las condiciones de temperatura, densidad y presión iniciales de los motores cohete.

Debido a estos factores se puede definir principalmente que la cámara de combustión debe tener un espacio para acomodar el propelente. Dicho espacio define la longitud de la cámara teniendo en cuenta que para 
todas las etapas el ángulo de convergencia a la garganta de los motores cohete es de $30^{\circ}$ con respecto al eje horizontal del motor. A continuación se puede observar el dimensionamiento de las cámaras de combustión para cada etapa [7]. Con el dimensionamiento de cada uno de los motores y las características del fluido en cada una de las partes del motor cohete se puede obtener la fuerza de empuje generada por cada motor en cada etapa.

TABLA 4 Comportamiento del propelente en la cámara de combustión.

\begin{tabular}{|l|l|l|l|}
\hline ETAPA & $T_{o}(\mathrm{~K})$ & $\rho_{o}\left(\mathrm{~kg} / \mathrm{m}^{3}\right.$ & $P_{o}(\mathrm{~Pa})$ \\
\hline I & 2889 & 2153 & 5066250 \\
\hline II & 2847 & 2153 & 2533125 \\
\hline III & 2847 & 2153 & 2533125 \\
\hline
\end{tabular}

TAB LA 5 Fuerza de empuje por etapa.

\begin{tabular}{|l|l|}
\hline ETAPA & $F(N)$ \\
\hline I & 552023 \\
\hline II & 56809 \\
\hline III & 7330 \\
\hline
\end{tabular}

Con el dimensionamiento de cada uno de los motores y las características del fluido en cada una de las partes del motor cohete se puede obtener la fuerza de empuje $F$ generada por cada motor en cada etapa.

Como se observa, el empuje para el motor de primera etapa es de aproximadamente 552023,1495 N lo que indica que es un empuje alto debido a que tiene que propulsar su propio peso además de la carga paga y los otros dos motores de segunda y tercera etapa. Como también se denota, los empujes de los motores de segunda y tercera etapa disminuyen debido a que la masa disminuye a medida que el cohete va quemando el propelente y desechando las etapas una vez estas dejen de producir empuje; necesitando una fuerza propulsiva menor para que el vehículo se acerque a la órbita donde deberá posicionar la carga útil que se transportara. Con este análisis se puede dar una aproximación de velocidad de cada etapa generando así una velocidad verdadera del cohete. La velocidad calculada anteriormente para el ducto propulsivo es la del fluido que va a impulsar el cohete, pero la velocidad verdadera es apreciada a continuación en la Tabla 6 la cual fue calculada teniendo en cuenta las masas iniciales y masas finales de cada una de las etapas [10].

TAB LA 6 Masas y velocidad verdadera del cohete para cada etapa.

\begin{tabular}{|l|l|l|l|l|l|}
\hline ETAPA & $m_{i}(\mathrm{~kg})$ & $m_{f}(\mathrm{~kg})$ & $m_{i} / m_{f}$ & $\Delta v(\mathrm{~m} / \mathrm{s})$ \\
\hline I & 65344 & 9548 & 0.146 & 4091 \\
\hline II & 9548 & 1601 & 0.168 & 3519 \\
\hline III & 1601 & 1401 & 0.125 & 4099 \\
\hline
\end{tabular}

TABLA 7 Espesores de las paredes de los motores para cada etapa.

\begin{tabular}{|l|l|}
\hline ETAPA & $t_{w}$ \\
\hline I & 0.026 \\
\hline II & 0.013 \\
\hline III & 0.013 \\
\hline
\end{tabular}

Para el cálculo de las masas se tiene en cuenta el espesor de las paredes de los motores y la cantidad de propelente usado. Posteriormente, se procede a escoger el material y a calcular los espesores de la cámara de combustión $t_{w}$ teniendo en cuenta el esfuerzo último $f t u$, el radio de la cámara $r_{o}$, la presión inicial de combustión $P_{o}$ que es la más alta y con un factor de seguridad $f_{s}$ de 3 escogido para el diseño [11]. La cual es calculada mediante la siguiente ecuación

$$
t_{w}=\frac{r_{o}+f_{s}+P_{o}}{f t u}
$$

A continuación en el gráfico 5 se observa una comparación de materiales teniendo en cuenta el espesor con respecto al esfuerzo último del material.

Como se puede observar en la Fig. 7, el material que tiene un mayor esfuerzo ultimo y que por ende arroja un espesor menor es el de la fibra de carbono que sería ideal para la construcción de los motores cohete para la disminución del peso, pero debido al alto costo del proceso de manufactura colombiano con este material se aconseja construir los motores cohete con Aluminio 7075-T6 debido a que los costos del material son menores y su fabricación en Colombia es viable tanto económica como técnicamente, y como se 
mencionaba anteriormente, el objetivo de diseñar el presente cohete, es elaborarlo totalmente por mano de obra colombiana [12]. Una vez escogido el Aluminio 7075-T6 como material para las cámaras de combustión, el espesor de la pared de la cámara para cada motor cohete teniendo en cuenta un factor de seguridad de 3 está dado en la Tabla 7. Se establece el mismo espesor tanto para la cámara de combustión como para la tobera en todos los motores.

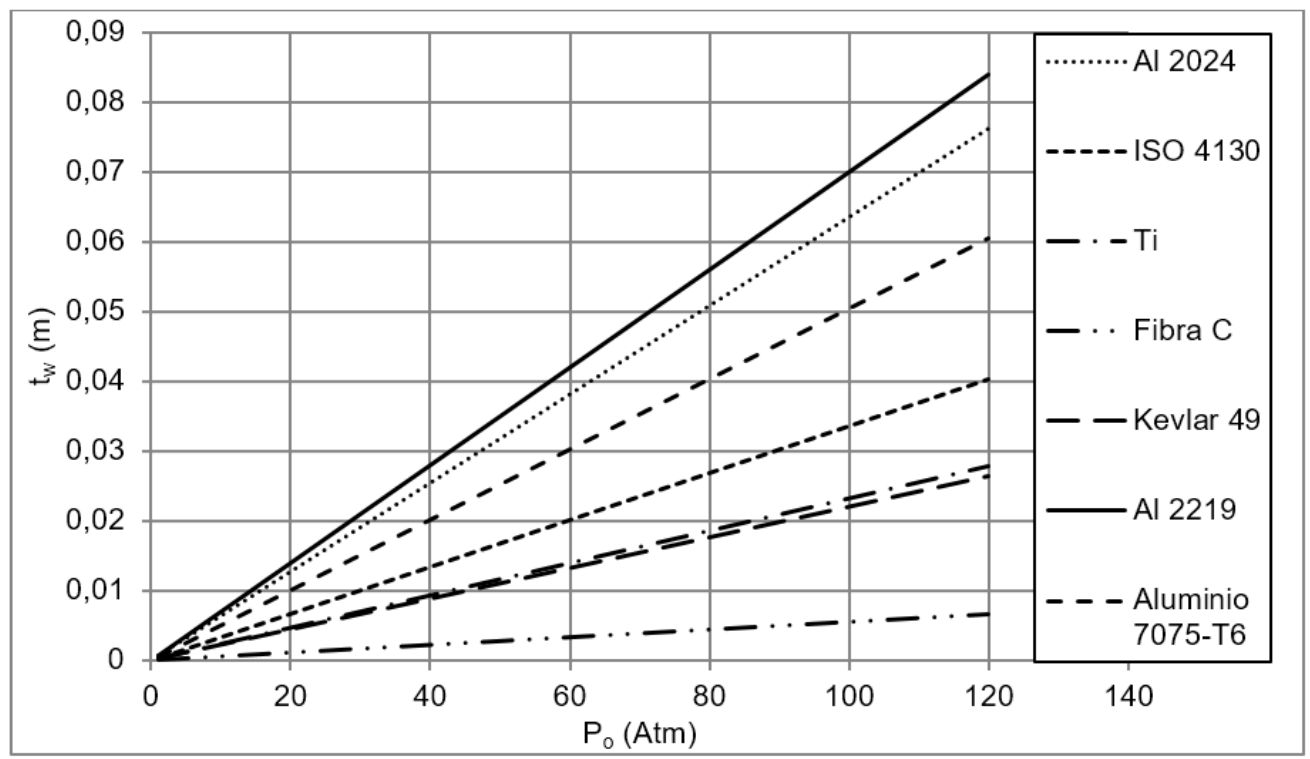

FIG. 7 Esfuerzo último de los materiales con relación a los espesores de la cámara.

\section{CONCLUSIONES}

El diseño conceptual elaborado presenta un incremento de masas respecto al cohete diseñado en la tesis de maestría de la Universidad Nacional de Colombia titulada "Estudio de la trayectoria de un cohete de tres etapas lanzado desde el territorio Colombiano" debido a que no toman en cuenta los espesores ni los materiales a usar en el cohete. El diseño conceptual del cohete de tres etapas es óptimo para efectuar la misión que es transportar $200 \mathrm{~kg}$ a una órbita baja de la Tierra (LEO), además es un cohete que puede ser fabricado en Colombia y ser lanzado desde el mismo territorio nacional debido a que todos los requerimientos de elaboración del diseño fueron esencialmente elaborados con las principales necesidades del país. Se puede destacar que los materiales y el dimensionamiento son parte de la comodidad de fabricación en Colombia lo que implica que este tipo de cohete va poder transportar una carga útil como son los satélites y los proyectos que se están elaborando en nuestra nación. El presente estudio muestra que el aluminio 7075-T6 es un material apropiado para la fabricación de motores cohete debido a que se ajusta a los parámetros de diseño estándar. Adicionalmente, la industria nacional cuenta con la capacidad de producción de este material.

\section{REFERENCIAS}

[1] PETER, M.A., Historia de la astronomía. Artículo de internet.

[2] Murcia, P. and J., "Estudio de la trayectoria de un cohete de tres etapas lanzado desde el territorio Colombiano," in Tesis de maestria. Bogotá DC.

[3] J. O. M. Piñeros and S. N. P. Laiton, "Diseño conceptual de un cohete sonda para toma de datos atmosféricos," TecnoESUFA: revista de tecnología aeronáutica, vol. 17, 2012. https://publicacionesfac.com/ index.php/TecnoESUFA/article/view/260. 
[4] J. Murcia, "Diseño conceptual de los motores cohete sólidos de primera etapa para un vehículo portador de satélites," Revista coningenio, 3: 198, vol. 207, 2010.

[5] J. O. M. Piñeros and S. A. G. Salcedo, "Diseño de los motores cohetes de propelente solido para el despegue asistido de un vehículo aéreo no tripulado (vant)," TecnoESUFA: revista de tecnología aeronáutica, vol. 17, 2012. https://publicacionesfac.com/index.php/TecnoESUFA/article/view/259.

[6] Huba, O., "Manuscrito de cátedra parte 2," in Universidad de Aachen Instituto de construcción liviana, p. 719.

[7] Fleeman, Eugene L, Tactical missile design, vol. 468. American Institute of Aeronautics and Astronautics Reston, VA, 2006.

[8] Sutton, G.P., Rocket Propulsion Elements. New York: JHON WILEY \& SONS. INC.

[9] Hibbeler, R., "Structural analysis 5Ed," in En R. Hibbeler, Structural analysis 5Ed, p. 771, United States: Prentice Hall.

[10] Griffin, M.D. and French, J.R. and Griffin, D. and French, J.R., "Space Vehicle Design," in En M, Blacksburg, Virginia: American Institute of Aernautics and Astronautics, inc.

[11] Fortescue, Peter and Swinerd, Graham and Stark, John, Spacecraft systems engineering. John Wiley \& Sons, 2011.

[12] MATWEB, "(s.f.). MATWEB." http://www. matweb.com. 